MPI-PhT/97-31

\title{
A Derivation of the Cyclic Form Factor Equation
}

\author{
Max R. Niedermaier \\ Max-Planck-Institut für Physik \\ (Werner Heisenberg Institut) \\ Föhringer Ring 6, D-80805 Munich, Germany
}

\begin{abstract}
A derivation of the cyclic form factor equation from quantum field theoretical principles is given; form factors being the matrix elements of a field operator between scattering states. The scattering states are constructed from Haag-Ruelle type interpolating fields with support in a 'comoving' Rindler spacetime. The cyclic form factor equation then arises from the KMS property of the modular operators $\Delta$ associated with the field algebras of these Rindler wedges. The derivation in particular shows that the equation holds in any massive $1+1 \mathrm{dim}$. relativistic QFT, regardless of its integrability.
\end{abstract}




\section{Introduction}

Form factors of a $1+1$ dim. massive quantum field theory (QFT) and modular structures in the sense of algebraic QFT are apparently unrelated concepts. Form factors are matrix elements of some field operator between an asymptotic multi-particle state and the physical vacuum. As such they are parametrized by a set of (initially real) rapidity variables $\theta_{j}$ in which they admit a meromorphic continuation and possibly by a set of internal quantum numbers $a_{j}, 1 \leq j \leq n$. We shall write $F_{a_{n} \ldots a_{1}}\left(\theta_{n}, \ldots, \theta_{1}\right)=\left\langle 0|\mathcal{O}| \theta_{n}, a_{n} ; \ldots ; \theta_{1}, a_{1}\right\rangle$, where $\mathcal{O}$ is a field operator (obtained by smearing a relativistic field, possibly nonlocal and charged with a test function) and each rapidity $\theta$ parametrizes an on-shell momentum in the usual way, $p_{0}=m \operatorname{ch} \theta, p_{1}=m \operatorname{sh} \theta$. In theories with a factorized scattering operator there exists a system of functional equations for these form factors, which entail that the Wightman functions built from them have all the required properties, and which in principle allow one to compute the former exactly. Knowing the form factors, the Wightman functions can be reconstructed through convergent series expansions, which arise from inserting a resolution of the identity in terms of scattering states. Truncating the series at a finite particle number provides a powerful solution technique that produces non-perturbative results difficult or impossible to obtain otherwise. The most innocently looking of these functional equations is the cyclic form factor equation, stating that

$$
F_{a_{n} \ldots a_{1}}\left(\theta_{n}, \ldots, \theta_{2}, \theta_{1}+2 \pi i\right)=\eta F_{a_{1} a_{n} \ldots a_{2}}\left(\theta_{1}, \theta_{n}, \ldots, \theta_{2}\right)
$$

where $\eta$ is a phase and the shift by $2 \pi i$ is understood in the sense of analytic continuation. Originally equation (1.1) was found in the context of the Sine-Gordon model [1] improving on earlier attempts to generalize Watsons equation [3]. Subsequently Smirnov promoted it to an axiom for the form factors of an integrable QFT, which together with the other equations implies locality [2]. The purpose of this paper is to give a derivation of equation (1.1) from quantum field theoretical principles. The derivation shows in particular that (1.1) holds in any massive $1+1 \mathrm{dim}$. relativistic QFT, regardless of its integrability. The crucial tools are the modular structures (in the sense of algebraic QFT) in a 'Rindler wedge' situation, where they have geometrical significance.

Modular structures in the context of von Neumann algebras are a pair of operators $(J, \Delta)$ that can be associated to any von Neumann algebra $\mathcal{M}$ with cyclic and separating vector $\Omega$. The latter means that there exists a Hilbert space $\mathcal{H}$ such that both $\mathcal{M} \Omega$ and $\mathcal{M}^{\prime} \Omega$ are dense subspaces of $\mathcal{H}$, where $\mathcal{M}^{\prime}$ is the commutant of $\mathcal{M}$. (The set and von Neumann 
algebra of all bounded operators on $\mathcal{H}$ commuting with $\mathcal{M}$.) The operator $J$ is an antiunitary involution with respect to the inner product on $\mathcal{H}$, and $\Delta$ is a positive selfadjoint (in general unbounded) operator. The defining relations for $(J, \Delta)$ are: $J \Delta^{1 / 2} X \Omega=$ $X^{*} \Omega$ for $X \in \mathcal{M}$ and $J \Delta^{-1 / 2} X^{\prime} \Omega=X^{\prime *} \Omega$ for $X^{\prime} \in \mathcal{M}^{\prime}$, where $J \Delta J=\Delta^{-1}$. The Tomita Takesaki theorem [7] states that $J \mathcal{M} J=\mathcal{M}^{\prime}$ and that for all real $\lambda$ the mapping $D_{\lambda}(X)=\Delta^{i \lambda} X \Delta^{-i \lambda}$ defines an automorphism group of both $\mathcal{M}$ and $\mathcal{M}^{\prime}$. From the defining relations one can deduce the following "KMS property" of $\Delta$, 6, , 7]

$$
(\Omega, Y \Delta X \Omega)=(\Omega, X Y \Omega), \quad X, Y \in \mathcal{M}
$$

Heuristically one can thus think of $\Delta$ as being an unbounded density operator for which the defining relations and (1.2) provide a substitute for the cyclic property of the trace.

In algebraic QFT one deals with a net of von Neumann algebras $\mathcal{M}(K)$ associated to bounded regions $K$ (double cones) of the Minkowski spacetime and (by the Reeh-Schlieder theorem) for each $\mathcal{M}(K)$ the vacuum provides a cyclic and separating vector. Hence the Tomita-Takesaki theory applies. The same holds when $K$ is replaced with a Rindler wedge, in which case the modular symmetries have geometrical significance. Basically $J$ acts as a reflection and exchanges the left and the right Rindler wedge and $-\frac{1}{2 \pi} \ln \Delta$ can be identified with the generator of Lorentz boosts along a direction that leaves the wedge invariant. Heuristically one can think of $\Delta$ as an unbounded operator implementing Lorentz boosts with purely imaginary parameter and $J$ as being related to the CPT operator. In the framework of the Wightman QFT the above result is essentially due to Bisognano and Wichmann [4], while in the more general algebraic setting an analogous $1+1$ dim. result has more recently been proved by Borchers [5].

In this context equation (1.1) is clearly reminiscent of the "KMS property" (1.2) of the modular operator $\Delta$. For an actual derivation of (1.1) based on (1.2) one has to deal with three aspects of the problem. First one has to make sure that the action of the modular operator is defined on (vectors generated by) appropriate operators localized in a wedge domain and having sharply peaked momentum transfer. Second one has to show that these operators generate the usual scattering states of a Minkowski space QFT. Third, in order to cover reasonably generic QFTs, soliton sectors should be taken into account. This is because in $1+1 \mathrm{dim}$. massive particles often have soliton properties, i.e. interpolate between inequivalent vacua, and excluding them asymptotic completeness cannot be expected to hold; see e.g. [13]. It is the combination of these aspects which renders the derivation of (1.1) technically a bit subtle. In the next section we describe the required general QFT framework in the presence of soliton sectors. In section 3 we 
discuss some aspects of a Haag-Ruelle type scattering theory tailored towards the use of modular structures. The derivation proper of (1.1) is given in section 4 .

\section{QFT framework including solitons}

Since equation (1.1) is a statement about matrix elements of scattering states, it is clear that the proper QFT framework for its derivation must ensure that the QFT under consideration has a well-behaved scattering theory. Apart from the set-up in which a Haag-Ruelle type scattering theory is formulated in higher dimensions, in $1+1 \mathrm{dim}$. this requires the inclusion of soliton sectors, because otherwise asymptotic completeness cannot be expected to hold. A model independent understanding of the appropriate QFT framework in the presence of soliton sectors was obtained only recently by Rehren and Müger [16, 18] based on early work by Fröhlich [13]. We shall adopt here a version of this framework suiting our purposes, the guideline being more simplicity rather than minimality of the assumptions. The paragraphs containing major assumptions on the QFT considered are numbered (1) - (6).

(1) The QFT is supposed to be described in terms of a net of local observables $K \rightarrow \mathcal{A}(K)$ satisfying isotony and locality [10]. For simplicity we also require covariance with respect to the action of the proper $1+1 \mathrm{dim}$. Poincaré group $P_{+}$. This means that there exists a representation of $P_{+}$by automorphisms $p \rightarrow \alpha_{p}$ such that $\alpha_{p} \mathcal{A}(K)=\mathcal{A}(p K)$. Elements $p=p(y, \lambda, r) \in P_{+}$can be parametrized by triples $(y, \lambda, r)$, where $y \in \mathbb{R}^{1,1}$ is a translation parameter, $\lambda \in \mathbb{R}$ is a boost parameter and $r \in\{ \pm 1\}$ is a sign. Our conventions are $p(y, \lambda, 1) x=y+x(\lambda)$, with $x^{0}(\lambda)=x^{0} \operatorname{ch} \lambda+x^{1} \operatorname{sh} \lambda, x^{1}(\lambda)=x^{0} \operatorname{sh} \lambda+x^{1} \operatorname{ch} \lambda$, and $p(0,0, \pm 1) x= \pm x$. The subgroup generated by $p(y, \lambda, 1)$ is the restricted Poincaré group, denoted by $P_{+}^{\uparrow}$. For arguments and indices referring to $p \in P_{+}$we will use the shorthands $y=p(y, 0,1), \lambda=p(0, \lambda, 1)$ and $r=p(0,0, r)$. The $C^{*}$-algebra associated with a double cone $K$ of $1+1 \mathrm{dim}$. Minkowski space is denoted by $\mathcal{A}(K)$ and is assumed to be a factor. In $1+1 \mathrm{dim}$. each double cone $K$ is an intersection of two translated wedges $K=(L+x) \cap(R+y)$, where $L=\left\{x \in \mathbb{R}^{1,1}|| x^{0} \mid<-x^{1}\right\}$ and $R=\left\{x \in \mathbb{R}^{1,1}|| x^{0} \mid<x^{1}\right\}$ are the left and right Rindler wedge. For an unbounded region $G$ let $\mathcal{A}(G)$ denote the algebra obtained by taking the normclosure $\left(C^{*}\right.$-inductive limit) of $\bigcup_{K \subset G} \mathcal{A}(G)$; in particular $\mathcal{A}=\mathcal{A}\left(\mathbb{R}^{1,1}\right)$ is the algebra of quasilocal observables. For any state $\omega$ over $\mathcal{A}(G)$ we write $\left(\mathcal{H}_{\omega}, \pi_{\omega}, \Omega\right)$ for the GNS triple of $\omega$ and denote by $\mathcal{M}_{\omega}(G)$ the von Neumann algebra 
$\pi(\mathcal{A}(G))^{\prime \prime}$, where the double prime denotes the weak closure in the $C^{*}$-algebra of bounded operators on $\mathcal{H}_{\omega}$. For the states $\omega$ of interest $\mathcal{H}_{\omega}$ carries a positive energy representation of the Poincaré group. If $P_{\omega}$ denotes the generator of the translation subgroup in $\mathcal{H}_{\omega}$ this means that its spectrum $\operatorname{Sp}\left(P_{\omega}\right)$ is contained in the closed forward lightcone (spectrum condition).

(2) Specifically we assume that the QFT under consideration has both massive 1-particle and massive vacuum states. These concepts are defined as follows [12]. A massive 1particle state is defined to a pure translation covariant state on $\mathcal{A}$ such that the spectrum $\operatorname{Sp}\left(P_{\omega}\right)$ on $\mathcal{H}_{\omega}$ consists of the mass shell $\left\{p \mid p_{0}>0, p^{2}=m^{2}\right\}$ and a subset of the continuum $\left\{p \mid p_{0}>0, p^{2}=(m+\mu)^{2}\right\}$, for some $\mu>0$. Similarly a massive vacuum state is defined, except that $\operatorname{Sp}\left(P_{\omega}\right)$ now consists of the value 0 and a subset of $\left\{p \mid p_{0}>0, p^{2} \geq \mu^{2}\right\}$, where $\mu>0$ is called the mass gap. The unitary equivalence class of irreducible GNS representations associated with a given massive 1-particle states is called a massive 1particle sector and will be denoted by $a$ or $\left[\omega_{a}\right]$. The set of massive 1-particle sectors is assumed to be finite and is denoted by $I$. Similarly massive vacuum sectors $\left[\omega_{\alpha}\right]$ are defined, of which there may be infinitely many. For these vacuum sectors we shall assume that they obey wedge duality, i.e.

$$
\mathcal{M}_{\alpha}(L+c)=\mathcal{M}_{\alpha}(R+c)^{\prime}, \quad \forall c \in \mathbb{R}^{1,1},
$$

where as usual the prime denotes the commutant in the algebra of bounded operators on the (separable) GNS Hilbert space. One can interpret $\mathcal{A}(L+c)$ as a weakly dense subalgebra of $\mathcal{M}_{\alpha}(L+c)$ and similarly for the right wedges. We do not require Haagduality.

If (2.1) is replaced with Haag duality, this is roughly also the $1+1$ dim. specialization of the set-up in which superselection sectors in $d+1 \mathrm{dim}$. in the sense of DHR and BF are discussed [11, 12, 10]. A peculiarity of $1+1 \mathrm{dim}$. is that non-trivial superselection sectors in this sense do not exist [16] (under certain conditions which are supposed to be satisfied in massive QFTs). The massive 1-particle states can however have soliton character, i.e. interpolate between two inequivalent vacua at positive or negative spacelike infinity. This is related to a topological speciality of $1+1 \mathrm{dim}$. Minkowski space: The spacelike complement of any double cone has two disconnected components, a left and a right component. Associated with any massive 1-particle state $\omega_{a}$ are therefore a pair of massive vacuum states $\omega_{\alpha_{L}}$ and $\omega_{\alpha_{R}}$ [14].

(3) Concerning the vacuum structure we assume that the different vacua arise (exclusively) 
from a spontaneously broken internal symmetry group $G$, as in [13]. For reasons that will become clear later we take the internal symmetry group to be abelian. More precisely $G$ is supposed to satisfy the following conditions: (G1) Elements $g \in G$ are $*$-automorphims of $\mathcal{A}$ preserving the net structure, i.e. $g(\mathcal{A}(K))=\mathcal{A}(K)$ for all double cones $K$. (G2) Elements $g \in G$ commute with the Poincaré group: $\alpha_{p} \circ g=g \circ \alpha_{p}, p \in P_{+}$. (G3) $G$ is abelian, finitely generated and has trivial second cohomology group. In concrete terms the latter means that every 2-cocycle $e: G \times G \rightarrow \mathbb{C}$ is a 2-coboundary, i.e.

$$
e\left(g_{2}, g_{3}\right) e\left(g_{1} g_{2}, g_{3}\right)^{*} e\left(g_{1}, g_{2} g_{3}\right) e\left(g_{1}, g_{2}\right)^{*}=1
$$

with $\left|e\left(g_{1}, g_{2}\right)\right|=1$ and $e(g, \mathbb{1})=1$ implies $e(g, h)=\lambda(g) \lambda(h) \lambda(g h)^{*}$, for some 1-cocycle $\lambda$ of $G$. Examples are the cyclic groups $\mathbb{Z}$ and $\mathbb{Z}_{N}, N>0$. (G4) The vacuum states $\omega_{\alpha}$ and $\omega_{\alpha} \circ g$ are unitarily inequivalent for all $g \neq 11$. As indicated, it is convenient to fix a reference vacuum state $\omega_{\alpha}$ and label all other vacua by group elements. In particular we shall write $\left(\mathcal{H}_{\alpha \circ g}, \pi_{\alpha \circ g}, \Omega\right)$ for the GNS triple of $\omega_{\alpha} \circ g$. The representation of the Poincaré group is the same in all vacuum representations and is denoted by $p \rightarrow U(p)$, with the shorthands $U(x)=U(p(x, 0,1))$, etc..

As described before massive 1-particle states will now interpolate between two such vacua at left and right spacelike infinity. In many situations one will be interested only in the interpolation properties of a state, not in its particle properties. This motivates to define kink states as follows: A state $\omega$ over $\mathcal{A}$ is called a kink state, interpolating between vacuum states $\omega_{\alpha} \circ g$ and $\omega_{\alpha} \circ h$, if it is a translation covariant state satisfying the spectrum condition and if it has the property

$$
\left.\left.\pi_{\omega}\right|_{\mathcal{A}(L)} \sim \pi_{\alpha \circ g}\right|_{\mathcal{A}(L)},\left.\left.\quad \quad \pi_{\omega}\right|_{\mathcal{A}(R)} \sim \pi_{\alpha \circ h}\right|_{\mathcal{A}(R)},
$$

where ' $\sim$ ' denotes unitary equivalence. Naturally a kink sector is an equivalence class $[\omega]$ of kink states. Note that massive 1-particle states are special kink states. Following Fröhlich [13] we next assume that (all) kink states can be constructed from vacuum states by means of suitable automorphisms of $\mathcal{A}$ whose existence is postulated.

(4) For any pair $(g, h) \in G \times G$ we assume that there exists a $*$-automorphism $\rho$ (a "kink automorphism of type $(g, h)$ ") enjoying the following properties:

( $\rho 1)$ There exists a bounded double cone $K$ such that

$$
\left.\rho\right|_{\mathcal{A}\left(K_{L}\right)}=\left.g\right|_{\mathcal{A}\left(K_{L}\right)} \quad \text { and }\left.\quad \rho\right|_{\mathcal{A}\left(K_{R}\right)}=\left.h\right|_{\mathcal{A}\left(K_{R}\right)},
$$


where $K_{L}$ and $K_{R}$ are the left and right spacelike complement of $K$, respectively. The region $K$ is called the interpolation region of $\rho$.

( $\rho 2) \rho$ commutes with the symmetries: $\rho g=g \rho$.

( $\rho 3)$ There exists a strongly contineous map $\gamma_{\rho}: P_{+}^{\uparrow} \rightarrow \mathcal{A}$ (a cocycle) such that

$$
\begin{aligned}
& \gamma_{\rho}(p) A \gamma_{\rho}(p)^{-1}=\left(\alpha_{p} \circ \rho \circ \alpha_{p^{-1}} \circ \rho^{-1}\right)(A), \quad A \in \mathcal{A}, \\
& \gamma_{\rho}\left(p_{2} p_{1}\right)=\alpha_{p_{2}}\left(\gamma_{\rho}\left(p_{1}\right)\right) \gamma_{\rho}\left(p_{2}\right), \\
& g \gamma_{\rho}(p)=\gamma_{\rho}(p), \quad \gamma_{g}(p)=\mathbb{1}, \quad g \in G .
\end{aligned}
$$

( $\rho 4)$ There exists a group homomorphism $G \times G \ni(11, h) \rightarrow \rho$, denoted by $h \rightarrow \rho_{h}$.

Let us add a few comments. The absence of non-trivial DHR sectors [16] implies that $\rho$ is determined by its type $(g, h)$ up to unitary equivalence. That is to say, if $\rho_{1}, \rho_{2}$ are two automorphisms of type $(g, h)$ then $\rho_{1} \rho_{2}^{-1}$ is an inner automorphism of $\mathcal{A}$. Condition $(\rho 4)$ thus says that out of each unitary equivalence class one can pick a representative such that $h \rightarrow \rho_{h}$ becomes a group homomorphism. Then ${ }_{g} \rho_{h}:=\rho_{h g^{-1}} g$ is a (preferred) kink automorphism of type $(g, h)$. The supplementary condition (2.5c) on the cocycles is only included for convenience; it could be relaxed and then follows from the other properties. Concerning $(\rho 1)$ one verifies that $\gamma_{\rho}(p), p=p(\lambda, x, 1)$ has interpolation region $x+p(\lambda, 0,1) K$, if $K$ is the interpolation region of $\rho$. Further one checks that the set of kink automorphisms forms a group with respect to composition. In particular the inverse of a kink automorphism of type $(g, h)$ is of type $\left(g^{-1}, h^{-1}\right)$ and has the same interpolation region. Parallel to the DHR case [11] one can show that two kink automorphisms commute if their interpolation regions are spacelike separated. Clearly a necessary condition for this to happen is that the group $G$ is abelian, which supplemented by $(\rho 2)$ also turns out to be sufficient [18]. If the interpolation regions of $\rho_{1}$ and $\rho_{2}$ are not spacelike separated, $\rho_{1} \rho_{2}$ and $\rho_{2} \rho_{1}$ are related by a unitary "statistics operator" as in the DHR case, $\rho_{1} \rho_{2}=\operatorname{Ad} \epsilon\left(\rho_{1}, \rho_{2}\right) \circ \rho_{2} \rho_{1}$. The latter is defined by separating the interpolation regions by means of unitary, gauge invariant charge transporters; by $2.5 \mathrm{c})$ the cocycles serve that purpose. It then follows that $\epsilon\left(\rho_{1}, \rho_{2}\right)$ depends at most on the orientation of the auxiliary spacelike separated regions employed in the separation process. In particular it satisfies $\epsilon\left(g_{1} \rho_{h_{1}}, g_{2} \rho_{h_{2}}\right)=\epsilon\left(\rho_{h_{1} g_{1}^{-1}}, \rho_{h_{2} g_{2}^{-1}}\right)$, and turns out always to be a complex phase. The statistics phase proper is defined as $\kappa_{\rho}=\epsilon(\rho, \rho)$ and obeys $\kappa_{\rho}=\kappa_{\rho^{-1}}=\kappa_{\rho \circ g}, \forall g \in G$. From 
( $\rho 4$ ), the trivial second cohomology of $G$, and the properties of the statistics operator one can show that there exists a choice of square roots $\sqrt{\kappa_{\rho}}$ such that

$$
\epsilon\left(\rho_{1}, \rho_{2}\right)=\frac{\sqrt{\kappa_{\rho_{1} \rho_{2}}}}{\sqrt{\kappa_{\rho_{1}}} \sqrt{\kappa_{\rho_{2}}}} .
$$

The main use of the kink automorphisms lies in the fact that they generate kink states from vacuum states. In detail, let $\rho$ be a kink automorphism of type $(g, h)$ and let $\omega_{\alpha}$ be a massive vacuum state. Then the state $\omega_{\alpha} \circ \rho$ is a kink state interpolating the vacuum sectors $\left[\omega_{\alpha} \circ g\right]$ and $\left[\omega_{\alpha} \circ h\right](*)$. Let us briefly comment on the proof of $(*)$. The fact that the state $\omega_{\alpha} \circ{ }_{g} \rho_{h}$ has the correct interpolation property is manifest. Its translation covariance follows from the translation part of the identity

$$
\begin{aligned}
& \left(\pi_{\alpha} \circ \rho \circ \alpha_{p}\right)(A)=U_{\rho}(p)\left(\pi_{\alpha} \circ \rho\right)(A) U_{\rho}(p)^{-1}, \quad \text { where } \\
& U_{\rho}(p):=U(p) \pi_{\alpha}\left(\gamma_{\rho}\left(p^{-1}\right)\right) .
\end{aligned}
$$

Here $p \rightarrow U_{\rho}(p)$ is the representation of the (restricted) Poincaré group in $\pi_{\alpha \circ \rho}:=\pi_{\alpha} \circ \rho$, which can thus be constructed from $U$ and the cocycle. To complete the proof of $(*)$ it remains to establish the spectrum condition, which is done in [13, 15].

Using the kink automorphisms therefore all superselection sectors, that is all of the relevant representations of $\mathcal{A}$ can be realized on a fixed reference Hilbert space, as in the DHR case [11, 12]. In detail, pick a reference vacuum state and let $\left(\mathcal{H}_{\alpha}, \pi_{\alpha}, \Omega\right)$ denote its GNS triple. For an interpolating automorphism $\rho$ consider the representation $\pi_{\alpha} \circ \rho$ with Hilbert space $\mathcal{H}_{\alpha \circ \rho}$. An automorphism $\hat{\rho}=\operatorname{Ad} V \circ \rho$ unitarily related to $\rho$ induces an unitarily equivalent representation $\pi \circ \hat{\rho}=\operatorname{Ad} W \circ(\pi \circ \rho), W=\pi(V)$ and vice versa. The space of cone-localized unitary intertwiners $V: \rho \rightarrow \hat{\rho}$ is denoted by $(\hat{\rho} \mid \rho)$. A generalized state is an equivalence class of pairs

$$
\left(\pi, \psi_{\alpha}\right) \sim\left(\operatorname{Ad} W \circ \pi, W \psi_{\alpha}\right), \quad W=\pi(V), V \in(\hat{\rho} \mid \rho)
$$

where $\psi_{\alpha} \in \mathcal{H}_{\alpha}$ and $\pi=\pi_{\alpha} \circ \rho$ for some interpolating automorphism $\rho$. We shall use $\left[\pi, \psi_{\alpha}\right]$ to denote the equivalence class generated by the pair $\left(\pi, \psi_{\alpha}\right)$. Each equivalence class $\left[\pi, \psi_{\alpha}\right]$ defines a state over $\mathcal{A}$ by means of the assignment

$$
\mathcal{A} \ni A \longrightarrow \frac{\left(\psi_{\alpha}, \pi(A) \psi_{\alpha}\right)}{\left(\psi_{\alpha}, \psi_{\alpha}\right)}
$$


The classes $\boldsymbol{\Omega}_{\alpha \circ g}:=\left[\pi_{\alpha} \circ g, \Omega\right]$ play the role of the vacua. The inner product of two pairs $(\pi, \psi)$ and $\left(\pi^{\prime}, \psi^{\prime}\right)$ is declared to vanish when $\pi$ and $\pi^{\prime}$ are not unitarily equivalent. Otherwise it is defined to be $\left(\psi, \psi^{\prime}\right)$ for representatives such that $\pi=\pi^{\prime}$. In particular the norm of $(\pi, \psi)$ is the norm of $\psi$. The space of all pairs $\left(\pi, \psi_{\alpha}\right)$ equipped with this inner product and norm is called the state bundle and is denoted by $\underline{\mathcal{H}}$.

The assumption $(\rho 4)$ allows one to choose a global section in this bundle. To see this let $\rho_{h}, h \in G$ be the collection of automorphisms forming a representation of $G$. As noted before, then ${ }_{g} \rho_{h}:=\rho_{h g^{-1}} g$ is an automorphism of type $(g, h)$ and any other of the same type is unitarily equivalent to it. Letting now $(g, h)$ run through $G \times G$, each sector is visited once and only once. If we denote by ${ }_{g} \mathcal{H}_{h}$ the Hilbert space of pairs $\left({ }_{g} \rho_{h}, \psi_{\alpha}\right)$, $\psi_{\alpha} \in \mathcal{H}_{\alpha}$, the direct sum $\bigoplus_{g, h}{ }^{\mathcal{H}}{ }_{h}$ provides a global section through the state bundle $\underline{\mathcal{H}}$.

Next we define an extension of the observable algebra acting irreducibly on $\underline{\mathcal{H}}$. It is obtained from pairs $(\rho, A)$ consisting of a kink automorphism and a quasilocal operator $A \in \mathcal{A}$. They act on pairs $\left(\pi, \psi_{\alpha}\right)$ by $(\rho, A)\left(\pi, \psi_{\alpha}\right)=\left(\pi \circ \rho, \pi(A) \psi_{\alpha}\right)$. The associated generalized state $\left[(\rho, A) \circ\left(\pi, \psi_{\alpha}\right)\right]$ defines a kink state over $\mathcal{A}$ by (2.9). The equivalence relation (2.8) induces a corresponding one $(\operatorname{Ad} V \circ \rho, V A) \sim(\rho, A)$ on the pairs $(\rho, A)$. The set of such pairs can be given the structure of an associative $*$-algebra with multiplication and $*$-operation given by

$$
\begin{aligned}
& \left(\rho_{2}, A_{2}\right)\left(\rho_{1}, A_{1}\right)=\left(\rho_{1} \rho_{2}, \rho_{1}\left(A_{2}\right) A_{1}\right) \\
& (\rho, A)^{*}=\left(\rho^{-1}, \rho^{-1}\left(\Phi^{*}\right)\right) \sim\left(\bar{\rho}, \bar{\rho}\left(A^{*}\right) V_{r}\right) .
\end{aligned}
$$

The automorphism $\bar{\rho}$ entering in the second line is defined by $\bar{\rho}=\alpha_{r} \circ \rho \circ \alpha_{r} \circ(g h)^{-1}$, where $\rho$ is of type $(g, h)$. Further $\alpha_{r}$ is the automorphism of $\mathcal{A}$ associated with $r=p(0,0,1) \in P_{+}$ and $V_{r}$ is a unitarity. $\bar{\rho}$ is of type $\left(g^{-1}, h^{-1}\right)$ and has interpolation region $-K$, if $K$ is the interpolation region of $\rho$. The former implies that $\bar{\rho}$ is unitarily related to $\rho^{-1}$, i.e. $\bar{\rho}=\operatorname{Ad} V_{r} \circ \rho^{-1}$, but has reflected interpolation region. In either version, the $*$-operation is compatible with the inner product on $\underline{\mathcal{H}}$. We shall refer to this algebra as "kink algebra" and denote it by $\mathcal{F}$. It can be given a net structure satisfying Poincaré covariance and isotony. The action of the (restricted) Poincaré group is

$$
(\rho, A) \stackrel{p}{\longrightarrow}\left(\rho, A_{\rho}(p)\right)=: \mathbf{U}(p)(\rho, A) \mathbf{U}(p)^{-1}, \quad \text { with } \quad A_{\rho}(p):=\gamma_{\rho}(p)^{*} \alpha_{p}(A)
$$

where $\gamma_{\rho}$ is a $P_{+}^{\uparrow}$-cocycle for $\rho$. As anticipated by the notation, the action (2.11) of the Poincaré group on pairs $(\rho, A)$ commutes with the composition law and the $*$-operation (2.10) due to the cocycle identity (2.5a). Concerning the localization properties, we say 
that a kink operator $(\rho, A)$ is localized in a double cone $K$ if there exists a representative $(\hat{\rho}, \widehat{A})$ in the unitarity equivalence class $(\operatorname{Ad} V \circ \rho, V A) \sim(\rho, A)$ such that $\hat{\rho}$ has interpolation region $K$ and $\widehat{A} \in \mathcal{A}(K)$. With this definition one shows that $\left(\rho, A_{\rho}(x)\right)$ is localized in $x+K$, if $(\rho, A)$ is localized in $K$, and that the product of two kink operators is localized in the smallest double cone containing the localization regions of the individual operators. The *-algebra of kink operators localized in $K$ is denoted by $\mathcal{F}(K)$, the subspace where $\rho$ is of type $(g, h)$ by ${ }_{g} \mathcal{F}_{h}(K)$.

Further the kink algebra carries two (mutually commuting) internal group actions. First, the original spontaneously broken symmetry, which is implemented unitarily on $\mathcal{F}$ via

$$
(\rho, \Phi) \stackrel{g}{\longrightarrow}(\rho, g(\Phi))=\mathbf{Q}_{g}^{-1}(\rho, \Phi) \mathbf{Q}_{g}=: g(\rho, \Phi)
$$

with $\mathbf{Q}_{g}=(g, \mathbb{1})$. In particular $\mathbf{Q}_{g}$ connects the different vacuum sectors in $\underline{\mathcal{H}}$ by $\mathrm{Q}_{g} \Omega_{\alpha}=\Omega_{\alpha \circ g}$. Second, there is an unbroken dual symmetry acting on $\mathcal{F}$ by $\left({ }_{g} \rho_{h}, \Phi\right) \rightarrow$ $\chi(g, h)\left({ }_{g} \rho_{h}, \Phi\right)$, where $\chi(g, h)$ is a character of $G \times G$. Both symmetries preserve the localization and commute with the $*$-operation and Poincaré transformations.

So far we have concentrated on the interpolation properties of the elements of the kink algebra $\mathcal{F}$. We now select those elements of $\mathcal{F}$ that generate interesting 1 -particle states from a vacuum $\Omega_{\alpha \circ g}$. First this requires $\rho$ to be such that $\omega_{\alpha} \circ g \rho$ is a massive 1-particle state. In addition $\Phi \in \mathcal{A}$ must be chosen such that the spectral support of this state is contained in the mass shell $\left\{p \mid p_{0}>0, p^{2}=m_{a}^{2}\right\}$. Finally it is natural to assume that $\Phi$ transforms irreducibly both under the action of the Lorentz group and under the action of $G$. Whence

$$
\mathbf{U}(\lambda) \mathbf{A} \mathbf{U}(\lambda)^{-1}=e^{s_{a} \lambda} \mathbf{A}, \quad \mathbf{Q}_{g}^{-1} \mathbf{A} \mathbf{Q}_{g}=\chi_{a}(g) \mathbf{A}
$$

where $s_{a} \in \mathbb{R}$ is called the spin of $\mathbf{A}$ and $\chi_{a} \in \widehat{G}$ the character of $\mathbf{A}$. As indicated we reserve an extra symbol $\mathbf{A}=(\rho, \Phi)$ for these elements of $\mathcal{F}$ and call them soliton operators, or 1-kink operators, of type $a=\left(g, h ; m_{a}, s_{a}, \chi_{a}\right)$. (The special case where $\rho$ actually interpolates between equivalent vacua, i.e. where $\mathbf{A}$ isn't a soliton operator proper, is included in this terminology.) The set of 1-kink operators $\mathbf{A}$ of type $a$ with interpolation region $K$ is denoted by $\mathcal{F}_{a}(K)$. By construction a soliton operator generates a 1-particle state from a vacuum sector. The 1-particle subspace of $\underline{\mathcal{H}}$ of type $a$ is denoted by $\mathcal{H}_{a}^{(1)}$. For the soliton operators one computes the following exchange relations [18]

$$
\mathbf{A} \mathbf{B}=\delta_{a b}( \pm) \mathbf{B} \mathbf{A}, \quad \pm\left(K_{a}-K_{b}\right) \succ 0
$$




$$
\begin{aligned}
& \delta_{a b}(+)=\epsilon\left(\rho_{a}, \rho_{b}\right) \chi_{b}^{*}\left(g_{a}\right) \chi_{a}\left(h_{b}\right), \\
& \delta_{a b}(-)=\epsilon\left(\rho_{b}, \rho_{a}\right)^{*} \chi_{b}^{*}\left(h_{a}\right) \chi_{a}\left(g_{b}\right) .
\end{aligned}
$$

Here ' $\succ$ ' denotes the partial ordering for double cones, i.e. $\widetilde{K} \succ K \Leftrightarrow \widetilde{K}-K \subset R$. The relations (2.13) and (2.14) clearly generalize to kink operators that are arbitrary products of soliton operators and which carry the induced quantum numbers.

(5) For the discussion of Spin-Statistics and the construction of a CPT operation it seems indispensable at present to assume that the kink algebra $\mathcal{F}$ is generated by non-local Wightman fields in the following sense: There are limits of operators $(\rho, A) \in \mathcal{F}(K)$ of pointlike lokalization, which upon translation give rise to non-local Wightman fields $\mathbf{F}(x)$, being unbounded operator-valued distributions. These fields then inherit the algebraic structures on $\mathcal{F}$ (group actions, multiplication and $*$-operation, interpolation properties, exchange relations etc.). In particular to each Wightman field $\mathbf{F}(x)$ a kink automorphism $\rho$ (with pointlike interpolation region) is associated and, after decomposition into irreducible components, also a group character $\chi$ and a Lorentz spin $s$. Cone-localized operators $\mathbf{F}$ can be recovered by averaging with appropriate test functions. We retain the previous terminology by saying that $\mathbf{F}$ is localized in $K$, if $\mathbf{F}$ arises from averaging a field $\mathbf{F}(x)$ with a test function supported in $K$ and if the kink automorphism associated with $\mathbf{F}$ can be chosen to have interpolation region $K$. In a slight abuse of notation we shall also write $K \rightarrow \mathcal{F}(K)$ for the local net (of unbounded operators) generated thereby, and continue to call the elements kink operators. In principle one can always switch to bounded counterparts of these operators and we assume that the net obtained thereby coincides with the previously defined kink algebra of bounded operators. For the purposes here the distinction between both descriptions is only essential for the construction of a CPT operation. In preparation of the latter, let $\mathbf{F}_{a}(x), \mathbf{F}_{b}(x)$ be two (Wightman) soliton fields of type $a, b$, respectively. Their two-point function obeys [18]

$$
\begin{aligned}
& \left(\mathbf{F}_{a}(x) \boldsymbol{\Omega}_{\alpha}, \mathbf{F}_{b}(y) \boldsymbol{\Omega}_{\alpha}\right)=\omega_{a} \omega_{b}^{*}\left(\mathbf{F}_{b}(-y)^{*} \boldsymbol{\Omega}_{\alpha}, \mathbf{F}_{a}(-x)^{*} \boldsymbol{\Omega}_{\alpha}\right), \quad(x-y)^{2}<0, \\
& \text { where } \quad \omega_{a}=\frac{e^{i \pi s}}{\sqrt{\kappa_{\rho}}} \chi(h)^{*}=e^{-i \pi s} \sqrt{\kappa_{\rho}} \chi(g)^{*} .
\end{aligned}
$$

Both expressions for $\omega_{a}$ coincide by the following spin-statistics relation [18]

$$
e^{2 \pi i s}=\kappa_{\rho} \chi\left(h g^{-1}\right) .
$$

This is to say, the spin of a soliton field of type $(g, h ; m, s, \chi)$ is determined up to an integer by $g h^{-1}$ and the character $\chi$. In order to get non-vanishing matrix elements in (2.15) $\rho_{a}$ 
and $\rho_{b}$ have to be of the same type $(g, h)$, in which case the phase in (2.15) only depends on the common unitary equivalence class $\rho \sim \operatorname{Ad} V \circ \rho$. In contrast, the character is not super

selected, i.e. $\chi_{a} \neq \chi_{b}, \chi_{a}, \chi_{b} \in \widehat{G}$ does not enforce the matrix element (2.15) to vanish. If the character were super selected only "neutral" operators of trivial character could have a non-vanishing vacuum expectation value and consequently the vacuum states $\omega_{\alpha \circ g}$ were all equal. From (2.15) one can anticipate the proper definition of the CPT operation, which for expositional reasons we defer to section 4 .

(6) Finally we assume 'completeness of the particle picture' in the following sense. First, there are sufficiently many soliton operators/fields in $\mathcal{F}_{a}(K)$ such that upon averaging the translated operators with rapidly decaying wave functions all of $\mathcal{H}_{a}^{(1)}$ can be generated from a vacuum sector. Second we assume the following version of asymptotic completeness. Given the collection of 1-particle Hilbert spaces $\mathcal{H}_{a}^{(1)}, a \in I$ one can apply a standard second quantization procedure to them, resulting in a Fock space. For the purposes here it is convenient to work with the free ('unsymmetrized') Fock space $F$. Off hand the Fock space $F$ is completely unrelated to the physical Hilbert space $\underline{\mathcal{H}}$. The Haag-Ruelle theory in this context provides a constructive way to isometrically embed two distinguished (proper or improper) subspaces $\underline{\mathcal{H}}^{\text {ex }}$, ex $=$ in/out of $\underline{\mathcal{H}}$ into $F$. We assume that the image in $F$ can be identified with subspaces of 'rapidity ordered' wave functions $F^{\mathrm{ex}}$, ex $=$ in/out; c.f. section 3, step 3. Since $F^{\text {in }}$ and $F^{\text {out }}$ are isometric this entails asymptotic completeness, i.e. $\underline{\mathcal{H}}^{\text {in }}=\underline{\mathcal{H}}^{\text {out }}$.

\section{Aspects of a Haag-Ruelle Scattering theory in $1+1 \mathrm{dim}$.}

Here we describe those aspects of a Haag-Ruelle type scattering theory in $1+1$ dimensions required for the derivation in section 4 . Compared to $3+1$ dimensions there are two technical complications. First, the convergence for $t \rightarrow \pm \infty$ of the states built from the multi-particle interpolating fields is only guaranteed for velocity ordered configurations. Second, the particle concept itself is more complicated due to the existence of solitons. As remarked before, the inclusion of soliton states is crucial for the discussion of scattering theory, because otherwise asymptotic completeness cannot be expected to hold. The assumption (5) is not needed here and the DHR description is used throughout this section.

The construction basically involves three steps: 
1. Construction of 1-particle interpolating fields.

2. Construction of multi-particle scattering states.

3. Verification that the norms of these states factorize, yielding isometric embeddings $\underline{\mathcal{H}}^{\mathrm{ex}} \rightarrow F$.

Tailored towards the use of geometric modular structures we wish to use ingredients localized in a wedge domain, which requires an approximation procedure. In the following we describe the so-adapted steps 1. - 3. consecutively.

1. Construction of the 1-particle interpolating fields: Let $(\rho, \Phi) \in \mathcal{F}_{a}(K)$ be a soliton operator of type $a$ and let $\widehat{\Phi}_{\rho}(p):=\int d^{2} x e^{-i p \cdot x} \Phi_{\rho}(x)$ be the Fourier transform of the translated operator $\Phi_{\rho}(x)$. We define the 1-particle interpolating field by

$$
\begin{array}{ll}
\mathbf{A}\left(f^{t} \mid \theta\right)=\left(\rho, A\left(f^{t} \mid \theta\right)\right), & A\left(f^{t} \mid \theta\right)=\int \frac{d^{2} p}{(2 \pi)^{2}} \widehat{f}^{t}(p) \widehat{\Phi}_{\rho}(p), \quad \text { where } \\
\widehat{f}^{t}(p)=\widehat{f}(p) e^{i\left(p_{0}-\omega\left(p_{1}\right)\right) t}, & \omega\left(p_{1}\right)=\sqrt{p_{1}^{2}+m_{a}^{2}} .
\end{array}
$$

Here $\widehat{f}(p)$ is a energy-momentum distribution with the following features: It is smooth (infinitely differentiable) with compact support in $\mathbb{R}^{1,1}$ and non-vanishing connected intersection with the mass hyperboloid $p^{2}=m_{a}^{2}$. For $\delta>0$ we define the velocity support of $f$ by

$$
v_{\delta}(f)=\left\{v(p):=p_{1} / \omega\left(p_{1}\right) \mid\|p-k\| \leq \delta, k \in \operatorname{supp}(\widehat{f})\right\}
$$

where $v(p)$ is the velocity with respect to the Lorentz frame determined by the $x^{0}$ coordinate. In $1+1 \mathrm{dim}$. it is convenient to use coordinates $p_{0}=\mu \cosh \theta, p_{1}=\mu \sinh \theta$ on the forward lightcone, in which case the velocity is parametrized by the rapidity $v(p)=\tanh \theta$. In particular $v_{\delta}(f)$ determines some closed rapidity interval. We shall refer to the center of this rapidity interval as the "average rapidity" $\hat{\theta}$. We also find it convenient to split the information contained in $\widehat{f}(p)$ into two parts: First an equivalence class of translated functions $\theta \rightarrow \widehat{f}(\mu \cosh (\theta-\lambda), \mu \sinh (\theta-\lambda))$ for some $\lambda \in \mathbb{R}$; and second the average rapidity $\widehat{\theta}$ of $\widehat{f}(p)$, which determines a unique member of this equivalence class. In the notation $A\left(f^{t} \mid \widehat{\theta}\right)$ adopted in (3.1), the first argument refers to the equivalence class and the second to the average rapidity. The advantage of this notation is that Lorentz boosts act on the fields (3.1) basically by shifting the average rapidity, i.e.

$$
\gamma_{\rho}(\lambda)^{*} \alpha_{\lambda}\left(A\left(f^{t} \mid \theta\right)\right)=e^{s_{a} \lambda} A\left(f^{t} \mid \theta+\lambda\right)
$$


Let us now address the localization properties of the 1-particle interpolating field $\mathbf{A}\left(f^{t} \mid \theta\right)$. In position space the expression (3.1) for $A\left(f^{t} \mid \theta\right)$ becomes

$$
\begin{aligned}
& A\left(f^{t} \mid \theta\right)=\int d^{2} x f^{t}(x) \Phi_{\rho}(x), \text { where } \\
& f^{t}(x)=\int \frac{d^{2} p}{(2 \pi)^{2}} \widehat{f}^{t}(p) e^{-i p \cdot x}=\int d^{2} y D^{t}(x-y) f(-y), \\
& D^{t}(x)=\int \frac{d^{2} p}{(2 \pi)^{2}} e^{i\left(p_{0}-\omega\left(p_{1}\right)\right) t} e^{-i p \cdot x} .
\end{aligned}
$$

Here $f$ is the Fourier transform of $\widehat{f}$ (but for notational simplicity $f^{t}$ is the Fourier transform of $\widehat{f}^{t}$ with sign reversed arguments). Since $\widehat{f}$ has compact support in momentum space, $f$ and $f^{t}$ will not have compact support in position space, but will only be of 'fast decrease'. In particular $A\left(f^{t} \mid \theta\right)$ is only a quasilocal field, not an element of any algebra $\mathcal{A}(K)$ associated with a bounded double cone $K$. With hindsight to the application of modular operators in a Rindler wedge situation we wish to approximate $A\left(f^{t} \mid \theta\right)$ by local fields. In preparation let us examine the decay properties of $f^{t}\left(x^{0}, x^{1}\right)$ in more detail. A standard integration by parts argument shows that it decays faster than any power of $\left|t-x^{0}\right|^{-1}$ for $\left|t-x^{0}\right| \rightarrow \infty$ with $x^{1}$ fixed. Similarly, for fixed $t$ it decays faster than any inverse power of $x^{1}$ for $x^{1} \rightarrow \infty$. Of particular interest is the limit along trajectories of the form $x^{0}=t, x^{1}=-v t$, with $v \notin v_{\delta}(f)$. Ruelle's lemma [9] states that $f^{t}(t,-v t)$ decays faster than any inverse power of $t$ for $|t| \rightarrow \infty$. This motivates to introduce compact regions

$$
G^{t, \delta}(f)=\left\{x \in \mathbb{R}^{1,1} \mid x^{0} \in[t-\delta, t+\delta], x^{1} \in-x^{0} v_{\delta}(f)\right\},
$$

whose spatial extension grows linearly in $|t|$. For a soliton operator $(\rho, \Phi) \in \mathcal{F}_{a}(K)$ then define

$$
\mathbf{A}^{\delta}\left(f^{t} \mid \theta\right)=\left(\rho, A^{\delta}\left(f^{t} \mid \theta\right)\right), \quad A^{\delta}\left(f^{t} \mid \theta\right)=\int_{G^{t, \delta}(f)} d^{2} x f^{t}(x) \Phi_{\rho}(x)
$$

and the bounded double cone

$$
K^{t, \delta}=\text { cone }\left(\bigcup_{x \in G^{t, \delta}(f)}(x+K)\right)
$$

where cone $(G)$ denotes the smallest double cone containing the set $G$. One can then show: (a) $\mathbf{A}^{\delta}\left(f^{t} \mid \theta\right)$ has interpolation region $K^{t, \delta}$. (b) The norm of the difference of the fields (3.4) and (3.6) is bounded by some rapidly decaying function $d(t)$, i.e. $\left\|A\left(f^{t} \mid \theta\right)-A^{\delta}\left(f^{t} \mid \theta\right)\right\|<$ $d(t)$.

\footnotetext{
${ }^{1} \mathrm{~A}$ quick check on the signs is via the stationary phase approximation.
} 
The proof of (a) can be found in [15]; we only add that the use of Haag duality can be avoided, consistent with our assumptions in section 2. In a slight abuse of notation we shall temporarily use $\left(\rho, A^{\delta}\left(f^{t} \mid \theta\right)\right)$ also to denote the representative $\left(\operatorname{Ad} V^{t} \circ \rho, V^{t} A^{\delta}\left(f^{t} \mid \theta\right)\right)$ ( $V^{t}$ a cone-localized unitarity) for which the automorphism has interpolation region $K^{t, \delta}$ and the operator is an element of $\mathcal{A}\left(K^{t, \delta}\right)$. Given Ruelle's lemma in the form

$$
\int_{R^{1,1} \backslash G^{t, \delta}(f)} d^{2} x f^{t}(x)<d(t)
$$

the proof of (b) amounts to

$$
\begin{aligned}
& \left\|A\left(f^{t} \mid \theta\right)-A^{\delta}\left(f^{t} \mid \theta\right)\right\|=\left\|\int_{R^{1,1} \backslash G^{t, \delta}(f)} d^{2} x f^{t}(x) \Phi_{\rho}(x)\right\| \\
& \leq\|\Phi\| \int_{R^{1,1} \backslash G^{t, \delta}(f)} d^{2} x f^{t}(x)<d(t)\|\Phi\| .
\end{aligned}
$$

2. Construction of multi-particle scattering states: Let $\left(\rho_{j}, \Phi_{j}\right) \in \mathcal{F}_{a_{j}}\left(K_{j}\right), 1 \leq j \leq n$, be a collection of soliton operators with interpolation regions $K_{j}$ to be specified later. Let $\mathbf{A}_{j}\left(f^{t} \mid \theta\right)=\left(\rho_{j}, A_{j}\left(f^{t} \mid \theta\right)\right)$ be the associated 1-particle interpolating fields and $\mathbf{A}_{j}^{\delta}\left(f^{t} \mid \theta\right)=$ $\left(\rho_{j}, A_{j}^{\delta}\left(f^{t} \mid \theta\right)\right)$ be the approximants (3.6). Using the composition law (2.10) the product fields can be computed, for which we introduce the shorthands

$$
\begin{aligned}
\mathbf{X}^{t, \delta} & =\mathbf{A}_{n}^{\delta}\left(f_{n}^{t} \mid \theta_{n}\right) \ldots \mathbf{A}_{1}^{\delta}\left(f_{1}^{t} \mid \theta_{1}\right)=:\left(\rho_{1} \ldots \rho_{n}, X^{t, \delta}\right) \\
\mathbf{X}^{t} & =\mathbf{A}_{n}\left(f_{n}^{t} \mid \theta_{n}\right) \ldots \mathbf{A}_{1}\left(f_{1}^{t} \mid \theta_{1}\right)=:\left(\rho_{1} \ldots \rho_{n}, X^{t}\right)
\end{aligned}
$$

for the restricted and unrestricted case, respectively. For the reference vacuum $\Omega_{\alpha}=$ $\left[\pi_{\alpha}, \Omega\right]$ consider the states $\mathbf{X}^{t} \boldsymbol{\Omega}_{\alpha}$ and $\mathbf{X}^{t, \delta} \boldsymbol{\Omega}_{\alpha}$. We wish to arrange the data on which these states depend such that for $t \rightarrow \infty$ they converge in norm to states in the physical Hilbert space $\underline{\mathcal{H}}$. The norm of pairs $(\rho, A)$ or $(\pi, \Psi)$ here is simply defined as the norm of the second entry of the pair. The convergence can be achieved by an appropriate choice of the localization regions $K_{j}$ of the 1-particle operators and the velocity supports $v_{\delta}\left(f_{j}\right)$ of the wave functions. The proper requirements are

$$
\begin{aligned}
& K_{n} \prec K_{n-1} \prec \ldots \prec K_{1}, \\
& v_{n}<v_{n-1}<\ldots<v_{1}, \quad \forall v_{j} \in v_{\delta}\left(f_{j}\right) .
\end{aligned}
$$

The states $\mathbf{X}^{t} \boldsymbol{\Omega}_{\alpha}$ with data (3.11) are the $1+1$ dim. version of Hepp-Ruelle "non-overlapping states". For the restricted fields $\mathbf{X}^{t, \delta}$ the condition $(3.11 \mathrm{~b})$ guarantees that the ordering 
(3.11a) translates into an ordering of the bounded interpolation regions (3.7)

$$
K_{n}^{t, \delta} \prec K_{n-1}^{t, \delta} \prec \ldots \prec K_{1}^{t, \delta}
$$

for large enough $t>0$. Further the spatial distance between these double cones tends to infinity as $t \rightarrow \infty$. On the other hand one has the multi-particle generalization of (3.9)

$$
\left\|\mathbf{X}^{t, \delta} \boldsymbol{\Omega}_{\alpha}-\mathbf{X}^{t} \boldsymbol{\Omega}_{\alpha}\right\|<d(t)
$$

Combining (3.12) and (3.13) one can follow the classic arguments [9, 11] to show that

$$
\left\|\frac{d}{d t} \mathbf{X}^{t} \mathbf{\Omega}_{\alpha}\right\|<d(t)
$$

for some rapidly decreasing function $d(t)$. A more detailed account can be found in section 6.3 of [15]. From (3.14) one concludes that the family of vectors $\mathbf{X}^{t} \boldsymbol{\Omega}_{\alpha}$ converges strongly for $t \rightarrow \infty$ to a vector in $\underline{\mathcal{H}}$, which is the searched for candidate for an $n$-particle 'out' scattering state. It turns out to depend only on the 1-particle input data; in particular it is easily checked to be independent of the choice of the Lorentz frame used. Further, by (3.13) the restricted interpolating fields generate the same scattering states. To adhere to the rapidity notation usually employed in the context of form factors, we shall describe the limits in terms of improper momentum eigenstates as follows

$$
\Psi^{\text {out }}:=\lim _{t \rightarrow \infty} \mathbf{X}^{t, \delta} \boldsymbol{\Omega}_{\alpha}=\lim _{t \rightarrow \infty} \mathbf{X}^{t} \boldsymbol{\Omega}_{\alpha}=: \int \frac{d^{n} \theta}{(4 \pi)^{n}} f_{n}\left(\theta_{n}\right) \ldots f_{1}\left(\theta_{1}\right)\left|\theta_{n}, a_{n} ; \ldots, \theta_{1}, a_{1}\right\rangle^{\text {out }}
$$

where $f_{j}(\theta)$ stands for $f_{j}\left(m_{a_{j}} \cosh \theta, m_{a_{j}} \sinh \theta\right), j=1, \ldots n$, and the massive 1-particle representations are $\pi_{a_{j}}=\pi_{\alpha} \circ \rho_{j}$. For simplicity we treat only 'out' scattering states here. For 'in' scattering states some of the ordering relations have to be reversed. Since we assume asymptotic completeness, it is convenient to treat them as CPT transforms of the 'out' states, as we shall do later.

3. Isometric embedding $\underline{\mathcal{H}}^{\text {out }} \rightarrow F$ : It remains to show that the norm of the limiting vectors (3.15) factorizes into a product of terms depending only on the 1-particle input data. As usual this follows from clustering, since the conditions (3.11) ensure that the spatial distances of the essential support regions (3.5) tend to infinity as $|t| \rightarrow \infty$, 9 , 11, 12. Details in the case at hand can be found in [15]. This factorization entails that the limiting states (3.15) can be identified with certain Fock space vectors having the same 
norm. In the momentum space description used before an $n$-particle vector is represented by a wave function $f^{(n)}\left(\theta_{n}, a_{n} ; \ldots ; \theta_{1}, a_{1}\right)$ with ordered and separated rapidities $\theta_{n}<\ldots<$ $\theta_{1}$, together with an assignment $\left(a_{n}, \ldots, a_{1}\right)$ to particle types. The space of sequences of such functions forms a subspace of the free Fock space $F$ (where no relations among the creation and annihilation operators are imposed) built from the 1-particle Hilbert spaces. Explicitly

$$
\begin{aligned}
& F^{\text {out }} \subset F=\bigoplus_{n=1}^{\infty} \bigoplus_{I^{n}} \mathcal{H}_{a_{n}}^{(1)} \otimes \ldots \otimes \mathcal{H}_{a_{1}}^{(1)}, \\
& F^{\text {out }}=\left\{\left(f^{(n)}\right)_{n \geq 0} \mid \operatorname{supp} f^{(n)} \in\left\{\theta \in \mathbb{R}^{n} \mid \theta_{n}<\ldots<\theta_{1}\right\}\right\} .
\end{aligned}
$$

The inner product on $F$ is inherited from the 1-particle sectors. The inner product on 1-particle states of type $a, b$ is

$$
\begin{aligned}
& \left(A\left(f_{1} \mid \theta_{1}\right) \Omega, A\left(f_{2} \mid \theta_{2}\right) \Omega\right)=\delta_{a, b} \int_{-\infty}^{\infty} \frac{d \theta}{4 \pi} f_{1}^{*}(\theta) f_{2}(\theta) \Longleftrightarrow \\
& { }^{\text {out }}\left\langle\theta_{1}, a \mid \theta_{2}, b\right\rangle^{\text {out }}=4 \pi \delta_{a b} \delta\left(\theta_{1}-\theta_{2}\right) .
\end{aligned}
$$

The isometric embedding $\underline{\mathcal{H}}^{\text {out }} \rightarrow F$ obtained thereby is a somewhat weaker result as in the usual Haag-Ruelle theory. The reason is that in $\underline{\mathcal{H}}^{\text {out }}$ additional relations among the state vectors exist, which result from the exchange relations (2.14) of the field operators (in coordinate space) used in their construction. Correspondingly the image $F^{\mathrm{sym}}$ of

$\underline{\mathcal{H}}^{\text {out }}$ in $F$ induced by $(3.15$ will consist of sequences of wave functions obeying certain 'symmetry' relations. In momentum space their explicit description may be cumbersome. Nevertheless one expects that these relations allow one to extend the domain of definition of an $n$-particle momentum space wave function from, say, the sector $\left\{\theta \in \mathbb{R}^{n} \mid \theta_{n}<\ldots<\right.$ $\left.\theta_{1}\right\}$ to all of $\mathbb{R}^{n}$, while preserving the norm. This is what the assumption in paragraph (6) of section 2 amounts to. In other words, we can view the exchange relations (2.14) as defining an isometry between $F^{\text {sym }}$ and $F^{\text {out }}$. For the purposes here only the final isometry between $\underline{\mathcal{H}}^{\text {out }}$ and $F^{\text {out }}$ matters.

\section{Cyclic form factor equation and modular structures}

After these lengthy preparations we now turn to the derivation proper of (1.1). The idea is to use the modular operators of a family of (right) wedge domains $R^{t}=c^{t}+R$ shifted 
along a path $t \rightarrow c^{t} \in \mathbb{R}^{1,1}$, such that the restricted interpolating fields at time $t$ have support in $R^{t}=c^{t}+R$ and the action of geometric modular operators is defined. In this way equation (1.1) arises from the "KMS property" (1.2) of the modular operator $\Delta$. As a guideline let us recall how (1.2) arises from the defining relations of $(J, \Delta)$. The latter are: $J \Delta^{1 / 2} X \Omega=X^{*} \Omega$ for $X \in \mathcal{M}$ and $J \Delta^{-1 / 2} X^{\prime} \Omega=X^{\prime *} \Omega$ for $X^{\prime} \in \mathcal{M}^{\prime}$, where $J \Delta J=\Delta^{-1}$. From this and the anti-unitarity of $J$ one obtains (1.2]) via [6, 7]

$$
\begin{aligned}
& (\Omega, Y \Delta X \Omega)=\left(\Delta^{1 / 2} Y^{*} \Omega, \Delta^{1 / 2} X \Omega\right) \\
& =\left(J Y \Omega, J X^{*} \Omega\right)=\left(X^{*} \Omega, Y \Omega\right)=(\Omega, X Y \Omega), \quad X, Y \in \mathcal{M} .
\end{aligned}
$$

The aim in the following is to transfer this computation to the situation at hand. To this end one first has to ensure that the $n$-particle interpolating fields have support in $R^{t}$ and that the action of $\Delta^{1 / 2}$ is defined on the vectors generated by them.

Let $\mathbf{A}_{j}^{\delta}\left(f_{j}^{t} \mid \theta_{j}\right), 1 \leq j \leq n$ be a collection of 1-particle interpolating fields with data satisfying (3.11). Let $\left(\rho_{j}, A_{j}^{\delta}\left(f_{j}^{t} \mid \theta_{j}\right)\right)$ be the representatives for which $\rho_{j}$ has interpolation region $K_{j}^{t, \delta}$ and $A_{j}^{\delta}\left(f_{j}^{t} \mid \theta_{j}\right) \in \mathcal{A}\left(K_{j}^{t, \delta}\right)$. For a suitably chosen $z^{t} \in \mathbb{R}^{1,1}$ we shall be interested in the analyticity properties in $\lambda$ of vectors of the form $U_{n}(\lambda) U_{n}\left(-z^{t}\right) \pi_{\alpha}\left(X^{t, \delta}\right) \Omega$, with $X^{t, \delta}$ as in (3.10). Writing this vector out explicitly, the kink representations $\pi_{j}:=\pi_{\alpha} \circ \rho_{1} \ldots \rho_{j}$ appear and we will use $U_{j}$ to denote $U_{\rho_{1} \ldots \rho_{j}}$ in (2.7). One finds

$$
\begin{aligned}
& U_{n}(\lambda) U_{n}\left(-z^{t}\right) \pi_{\alpha}\left(X^{t, \delta}\right) \Omega \\
& =e^{\left(s_{a_{n}}+\ldots+s_{a_{1}}\right) \lambda} \int_{G_{n}^{t}} d^{2} y_{n} \ldots \int_{G_{1}^{t}} d^{2} y_{1} f_{n}^{t}\left(y_{n}\right) \ldots f_{1}^{t}\left(y_{1}\right) U_{n}\left(y_{n}(\lambda)-z^{t}(\lambda)\right) \times \\
& \times \pi_{n-1}\left(\Phi_{n}\right) U_{n-1}\left(y_{n-1}(\lambda)-y_{n}(\lambda)\right) \ldots \pi_{1}\left(\Phi_{2}\right) U_{1}\left(y_{1}(\lambda)-y_{2}(\lambda)\right) \pi_{\alpha}\left(\Phi_{1}\right) \Omega .
\end{aligned}
$$

Here $z^{t} \in \mathbb{R}^{1,1}$ is chosen such that $-z^{t}+G_{n}^{t} \subset R$ and to simplify the notation we wrote $G_{j}^{t}$ for $G^{t, \delta}\left(f_{j}\right), 1 \leq j \leq n$. The guideline to determine the analyticity properties of (4.2) is the following simple fact. Let $p \rightarrow U(p)$ be a strongly continous unitary representation of $P_{+}^{\uparrow}$ on a separable Hilbert space obeying the spectrum condition. Consider $U(x(\lambda))=$ $U(\lambda) U(x) U(\lambda)^{-1}$ for $x \in \mathbb{R}^{1,1}$, with the notation $x^{0}(\lambda)=x^{0} \operatorname{ch} \lambda+x^{1} \operatorname{sh} \lambda, x^{1}(\lambda)=x^{0} \operatorname{sh} \lambda+$ $x^{1} \operatorname{ch} \lambda$. Then

$$
\lambda \rightarrow U(x(\lambda)) \text { is analytic in }\left\{\begin{aligned}
0<\operatorname{Im} \lambda<\pi, & \text { if } x \in R, \\
-\pi<\operatorname{Im} \lambda<0, & \text { if } x \in L .
\end{aligned}\right.
$$

Further $U(x(\lambda))$ is a bounded operator in these strips. Applied to the vector (4.2) one sees that the dependence on $\lambda$ is analytic in the strip $0<\operatorname{Im} \lambda<\pi$. Indeed, since the 
spatial distance between the regions $G_{j}^{t}$ increases with $t$, there exists a $t_{0}>0$ such that $\operatorname{cone}\left(G_{n}^{t}\right) \prec \ldots \prec \operatorname{cone}\left(G_{1}^{t}\right)$, for $t \geq t_{0}$, which implies $y_{j}-y_{j+1} \in R$ for $j=1, \ldots, n-1$ with $y_{j} \in G_{j}^{t}$. For the argument of $U_{n}$ the condition $y_{n}-z^{t} \in R$ holds by definition of $z^{t}$. In summary, we found that for a suitably chosen $z^{t} \in \mathbb{R}^{1,1}$ the support regions $G_{j}^{t}=$ $G^{t, \delta}\left(f_{j}\right)$ are contained in a shifted right wedge domain $z^{t}+R$, for $t>t_{0}$. In this shifted wedge $U_{n, z^{t}}(\lambda):=U_{n}\left(z^{t}\right) U_{n}(\lambda) U_{n}\left(-z^{t}\right)$ plays the role of the Lorentz boost generator and acts consistently on the vector $\pi_{\alpha}\left(X^{t, \delta}\right) \Omega$ for $0 \leq \operatorname{Im} \lambda \leq \pi$. The localization regions $K_{j}^{t, \delta}$ of the 1-particle interpolating fields are not necessarily contained in $z^{t}+R$. However, since they are likewise compact regions, related to $G_{j}^{t}$ by $(3.7)$, one can find a $c^{t} \in \mathbb{R}^{1,1}$ (timelike and future-pointing) and $t_{1} \geq t_{0}$ such that $K_{j}^{t, \delta} \subset c^{t}+R$, for all $t>t_{1}$. With this definition of $R^{t}:=c^{t}+R$ one has $A_{j}^{\delta}\left(f_{j}^{t} \mid \theta_{j}\right) \in \mathcal{A}\left(K_{j}^{t}\right) \subset \mathcal{A}\left(R^{t}\right)$. It is easy to see that such localization properties are preserved under composition of kink operators. For the multiparticle interpolating fields (3.10) one can thus choose representatives $\rho_{X}=$ $\operatorname{Ad} V^{t} \rho_{1} \ldots \rho_{n}\left(V^{t}\right.$ a cone-localized unitarity) having interpolation region cone $\left(K_{1}^{t, \delta} \cup \ldots \cup\right.$ $\left.K_{n}^{t, \delta}\right)$ and $X\left(R^{t}\right):=V^{t} X^{t, \delta} \in \mathcal{A}\left(R^{t}\right)$. Having ensured that such a choice of representatives is possible, the outcome of the previous discussion is conveniently recast in terms of kink operators and generalized states. Set

$$
\mathbf{U}_{t}(\lambda):=\mathbf{U}\left(c^{t}\right) \mathbf{U}(\lambda) \mathbf{U}\left(-c^{t}\right), \quad \boldsymbol{\Delta}_{R}^{s}:=\mathbf{U}_{t}(2 \pi i s), \quad \boldsymbol{\Delta}_{L}^{s}:=\mathbf{U}_{t}(-2 \pi i s), \quad s>0 .
$$

\section{Proposition 1}

(a) Let $\mathbf{A}_{j}^{\delta}\left(f_{j}^{t} \mid \theta_{j}\right), 1 \leq j \leq n$, be restricted 1-particle interpolating fields with data satisfying (3.11). Then there exist wedge domains $R^{t}=c^{t}+R$ and $t_{1}>0$ such that the restricted $n$-particle interpolating field

$$
\mathbf{X}\left(R^{t}\right):=\mathbf{A}_{n}^{\delta}\left(f_{n}^{t} \mid \theta_{n}\right) \ldots \mathbf{A}_{1}^{\delta}\left(f_{1}^{t} \mid \theta_{1}\right)=\left(\rho_{X}, X\left(R^{t}\right)\right)
$$

has bounded interpolation region in $R^{t}$ for all $t>t_{1}$. Symbolically $\mathbf{X}\left(R^{t}\right) \in \mathcal{F}\left(R^{t}\right)$.

(b) $\boldsymbol{\Delta}_{R}^{s}$ is a positive densely defined operator on $\mathcal{F}\left(R^{t}\right) \boldsymbol{\Omega}_{\alpha}$, for all $0 \leq s \leq 1 / 2$.

(c) The generalized states $\mathbf{X}\left(R^{t}\right) \boldsymbol{\Omega}_{\alpha}$ converge strongly to scattering states in $\underline{\mathcal{H}}$ for $t \rightarrow \infty$.

Until here the assumption (5) did not enter. Now we employ it to construct a CPT operation on $\mathcal{F}$ in its Wightman version. Recall the notation $\mathbf{Q}_{g}=(g, \mathbb{1})$ and let $\mathbf{Q}$ be the unitary involution on $\underline{\mathcal{H}}$, acting like $\mathbf{Q}_{(g h)^{-1}}$ on the sector ${ }_{g} \mathcal{H}_{h}$. Define an operator $\boldsymbol{\Theta}$ 
on $\underline{\mathcal{H}}$ and $\operatorname{Ad} \Theta$ on $\mathcal{F}$ by

$$
\boldsymbol{\Theta F}(x) \boldsymbol{\Omega}_{\alpha}=\omega_{a} \mathbf{Q} \mathbf{F}(-x)^{*} \boldsymbol{\Omega}_{\alpha}, \quad \boldsymbol{\Theta F}(x) \boldsymbol{\Theta}=\omega_{a} \mathbf{Q}_{g h} \mathbf{F}(-x)^{*},
$$

where in the second equation $\mathbf{F}(x)$ is of type $(g, h)$ and hence $\mathbf{Q}_{g h} \mathbf{F}(-x)^{*}$ is of type $(h, g)$. Then $\Theta$ has the following properties:

\section{Proposition 2}

(a) $\operatorname{Ad} \boldsymbol{\Theta}$ is an antilinear $*$-automorphism of $\mathcal{F}$ and an involution. Further $\boldsymbol{\Theta}$ is antiunitary w.r.t. the inner product on $\underline{\mathcal{H}}$.

(b) The following commutation relations hold

$$
\begin{aligned}
\Theta \mathbf{U}( \pm i \pi) & =\mathbf{U}(\mp i \pi) \Theta, & \boldsymbol{\Theta Q}_{g} & =\mathbf{Q}_{g} \Theta \\
\Theta \mathbf{U}(\lambda) & =\mathbf{U}(\lambda) \Theta, & \Theta \mathbf{U}(x) & =\mathbf{U}(-x) \Theta
\end{aligned}
$$

(c) $\boldsymbol{\Theta} \mathcal{F}(R) \Theta=\mathcal{F}(L)$ and vice versa.

The proof can be adapted from Rehren [18. This CPT operator arises, up to a unitary factor, from the polar decomposition of the following Tomita operators

$$
\begin{array}{ll}
\mathbf{S}_{+} \mathbf{F} \boldsymbol{\Omega}_{\alpha}=\mathbf{Q} h\left(\mathbf{F}^{*}\right) \boldsymbol{\Omega}_{\alpha}, & \mathbf{F} \in{ }_{g} \mathcal{F}_{h}(R), \\
\mathbf{S}_{-} \mathbf{F} \boldsymbol{\Omega}_{\alpha}=\mathbf{Q} g\left(\mathbf{F}^{*}\right) \boldsymbol{\Omega}_{\alpha}, & \mathbf{F} \in{ }_{g} \mathcal{F}_{h}(L) .
\end{array}
$$

In fact, the closures of the operators (4.8) can be seen to have adjoints related by $\left(\mathbf{S}_{ \pm}\right)^{*}=$ $\kappa^{ \pm 1} \mathbf{S}_{\mp}$ and to admit polar decompositions

$$
\mathbf{S}_{ \pm}=\mathbf{J}_{ \pm} \mathbf{U}( \pm i \pi), \quad \text { with } \quad \mathbf{J}_{ \pm}=\sqrt{\kappa}^{ \pm 1} \Theta
$$

Here the unitary operator $\sqrt{\kappa}$ is declared to act by multiplication with $\sqrt{\kappa_{\rho}}$ on $\mathcal{H}_{\alpha \circ \rho}$. The origin of the unitary factor $\sqrt{\kappa}^{ \pm 1}$ can be understood from the relations

$$
\begin{array}{ll}
\boldsymbol{\Theta U}(i \pi) \mathbf{F} \boldsymbol{\Omega}_{\alpha}=\sqrt{\kappa}^{-1} \mathbf{Q} h\left(\mathbf{F}^{*}\right) \boldsymbol{\Omega}_{\alpha}, & \mathbf{F} \in{ }_{g} \mathcal{F}_{h}(R), \\
\boldsymbol{\Theta} \mathbf{U}(-i \pi) \mathbf{F} \boldsymbol{\Omega}_{\alpha}=\sqrt{\kappa} \mathbf{Q} g\left(\mathbf{F}^{*}\right) \boldsymbol{\Omega}_{\alpha}, & \mathbf{F} \in{ }_{g} \mathcal{F}_{h}(L) .
\end{array}
$$

Next we show that the CPT operation declared via (4.6) on the kink operators induces a CPT operation on scattering states having all the required properties. The CPT conjugate 
of a 1-particle (Wightman) interpolating field of type $(g, h)$ naturally is

$$
\begin{aligned}
& \boldsymbol{\Theta}\left(\rho, A^{\delta}\left(f^{t} \mid \theta\right)\right) \boldsymbol{\Theta}=\left(j(\rho), A^{\delta, C P T}\left(f^{*-t} \mid \bar{\theta}\right)\right), \quad \text { where } \\
& j(\rho)=\bar{\rho} g h, \quad A_{\mathrm{CPT}}^{\delta}\left(f^{*-t} \mid \bar{\theta}\right)=\omega_{a} \int_{G^{-t, \delta}\left(f^{*}\right)} d^{2} y f^{*-t}(y) \Phi^{*}(y) .
\end{aligned}
$$

We have displayed the representatives localized in $-K^{t, \delta}=-G^{t, \delta}$ and rewrote the operator such that the time reversal is manifest. The complex conjugate $f^{*}$ of $f$ plays the role of the charge conjugate wave function, whose average rapidity is denoted by $\bar{\theta}$. Of course the spacetime reflection here is with respect to the origin of the chosen coordinate system and exchanges $R$ with $L$, rather than the 'comoving' wedge domains $R^{t}=c^{t}+R$ and $c^{t}+L=: L^{t}$. A CPT operation doing the latter is

$$
\boldsymbol{\Theta}_{t}:=\mathbf{U}\left(c^{t}\right) \boldsymbol{\Theta} \mathbf{U}\left(-c^{t}\right)
$$

Let then $\mathbf{X}\left(R^{t}\right)$ be an $n$-particle interpolating field as in (4.5) and consider its CPT conjugate

$$
\boldsymbol{\Theta}_{t} \mathbf{X}\left(R^{t}\right) \boldsymbol{\Theta}_{t}=\left(j\left(\rho_{n}\right) \ldots j\left(\rho_{1}\right), X\left(R^{t}\right)_{\mathrm{CPT}}\right)=: \mathbf{X}\left(R^{t}\right)_{\mathrm{CPT}}
$$

One easily sees that there exist representatives, displayed in the middle term, for which $X\left(R^{t}\right)_{\mathrm{CPT}} \in \mathcal{A}\left(L^{t}\right)$ and $j\left(\rho_{1}\right) \ldots j\left(\rho_{n}\right)$ has bounded interpolation region in $L^{t}:=c^{t}+L$ for $t<-t_{1}$. As before one can use them to study the analyticity properties in $\lambda$ of the Lorentz boosted state $\mathbf{U}(\lambda) \mathbf{U}\left(-c^{t}\right) \mathbf{X}\left(R^{t}\right)_{\mathrm{CPT}} \boldsymbol{\Omega}_{\alpha}$ as in (4.2). With the data for $\mathbf{X}\left(R^{t}\right)$ as in proposition 1 , the dependence on $\lambda$ is found to be analytic in the strip $-\pi<\operatorname{Im} \lambda<0$. It follows that the action of $\Delta_{L}^{s}, 0 \leq s \leq 1 / 2$ is defined on $\mathbf{X}\left(R^{t}\right)_{\mathrm{CPT}}$. Since the latter generate $\mathcal{F}\left(L^{t}\right)$ one concludes that

$$
\Theta_{t} \mathcal{F}\left(R^{t}\right) \Theta_{t}=\mathcal{F}\left(L^{t}\right), \quad \Theta_{t} \Delta_{L}^{s} \Theta_{t}=\Delta_{R}^{s}, \quad 0 \leq s \leq 1 / 2, \quad \text { on } \mathcal{F}\left(R^{t}\right) \boldsymbol{\Omega}_{\alpha}
$$

Further

$$
\begin{array}{ll}
\mathbf{S}_{R} \mathbf{X} \boldsymbol{\Omega}_{\alpha}=\mathbf{Q} h\left(\mathbf{X}^{*}\right) \boldsymbol{\Omega}_{\alpha}, & \mathbf{X} \in{ }_{g} \mathcal{F}_{h}\left(R^{t}\right), \quad \text { with } \mathbf{S}_{R}=\sqrt{\kappa} \boldsymbol{\Theta}_{t} \boldsymbol{\Delta}_{R}^{1 / 2} \\
\mathbf{S}_{L} \mathbf{X} \boldsymbol{\Omega}_{\alpha}=\mathbf{Q} g\left(\mathbf{X}^{*}\right) \boldsymbol{\Omega}_{\alpha}, & \mathbf{X} \in{ }_{g} \mathcal{F}_{h}\left(L^{t}\right), \quad \text { with } \mathbf{S}_{L}=\sqrt{\kappa}{ }^{-1} \boldsymbol{\Theta}_{t} \boldsymbol{\Delta}_{L}^{1 / 2}
\end{array}
$$

In particular the state $\mathbf{X}\left(R^{t}\right)_{\mathrm{CPT}} \Omega_{\alpha}$ converges strongly to a scattering state in $\underline{\mathcal{H}}$ for $t \rightarrow \infty$. On the improper scattering states (3.15) the following CPT operation is induced

$$
\mathbf{J}\left|\theta_{n}, a_{n} ; \ldots ; \theta_{1}, a_{1}\right\rangle^{\text {out }}=\left|\bar{\theta}_{1}, \bar{a}_{1} ; \ldots ; \bar{\theta}_{n}, \bar{a}_{n}\right\rangle^{\text {in }}=\left|\bar{\theta}_{n}, j\left(a_{n}\right) ; \ldots ; \bar{\theta}_{1}, j\left(a_{1}\right)\right\rangle^{\text {in }}
$$


Here $a_{k}, \bar{a}_{k}$ and $j\left(a_{k}\right)$ refer to the massive 1-particle representations $\pi_{\alpha} \circ \rho_{k}, \pi_{\alpha} \circ \bar{\rho}_{k}$ and $\pi_{\alpha} \circ j\left(\rho_{k}\right)$, respectively. Further $\theta$ and $\bar{\theta}$ are the average rapidities of a momentum space wave function $\widehat{f}$ and its complex conjugate, respectively. From (4.16) one readily checks that $\mathbf{J}$ has all the familiar properties of a CPT operation on scattering states. In particular it leaves the scattering operator invariant $\mathbf{J} \mathcal{S} \mathbf{J}=\mathcal{S}^{-1}$ and the scattering operator $\mathcal{S}$ itself can be written as a product of $\mathbf{J}$ and the free CPT operator on the Fock space; see also 117.

Having all these ingredients at our disposal we can eventually transfer the computation (4.1) to the case at hand. Introduce generalized operators $\mathbf{X}\left(R^{t}\right), \mathbf{Y}\left(R^{t}\right)$ by

$$
\begin{aligned}
& \mathbf{X}\left(R^{t}\right):=\mathbf{A}_{n}^{\delta}\left(f_{n}^{t} \mid \theta_{n}\right) \ldots \mathbf{A}_{n-k+1}^{\delta}\left(f_{n-k+1}^{t} \mid \theta_{n-k+1}\right)=\left(\rho_{X}, X\left(R^{t}\right)\right) \\
& \mathbf{Y}\left(R^{t}\right):=\mathbf{A}_{n-k}^{\delta}\left(f_{n-k}^{t} \mid \theta_{n-k}\right) \ldots \mathbf{A}_{1}^{\delta}\left(f_{1}^{t} \mid \theta_{1}\right)=\left(\rho_{Y}, Y\left(R^{t}\right)\right)
\end{aligned}
$$

where the data $f_{j}$ and $K_{j}, 1 \leq j \leq n$ are as in proposition 1 and the terms on the right denote the representatives with interpolation region $K_{X}:=\operatorname{cone}\left(K_{n}^{t, \delta} \cup \ldots \cup K_{n-k+1}^{t, \delta}\right)$ and $K_{Y}:=\operatorname{cone}\left(K_{n-k}^{t, \delta} \cup \ldots \cup K_{1}^{t, \delta}\right)$, respectively. Further let $\mathbf{O}=\left(\rho_{O}, \mathcal{O}\right)$ be a kink operator and choose $d^{t} \in \mathbb{R}^{1,1}$ such that the translated operator $\mathbf{O}\left(d^{t}\right):=\mathbf{U}\left(d^{t}\right) \mathbf{O U}\left(-d^{t}\right)$ has interpolation region $K_{O}$ satisfying $K_{X} \prec K_{O} \prec K_{Y}$, for large $t$. In order to have nonvanishing matrix elements of the form required, these operators have to satisfy an appropriate 'charge balance' condition. Explicitly, for some $k \in G$ we assume that

$$
\rho_{Y} \rho_{O} \rho_{X}=k
$$

and write $\boldsymbol{\Omega}_{\beta}:=\boldsymbol{\Omega}_{\alpha \circ k}$. Further we abbreviate momentarily $\mathbf{X}=\mathbf{X}\left(R^{t}\right), \mathbf{Y}=\mathbf{Y}\left(R^{t}\right)$. Using (4.18) one verifies that all the matrix elements in the following chain of equalities are well-defined

$$
\begin{aligned}
& \left(\boldsymbol{\Delta}_{R}^{1 / 2} \mathbf{Y}^{*} \mathbf{O}^{*}\left(d^{t}\right) \boldsymbol{\Omega}_{\beta}, \boldsymbol{\Delta}_{R}^{1 / 2} \mathbf{X} \boldsymbol{\Omega}_{\alpha}\right)=\left(\boldsymbol{\Theta}_{t} \sqrt{\kappa}^{-1} \mathbf{S}_{R} \mathbf{Y}^{*} \mathbf{O}^{*}\left(d^{t}\right) \boldsymbol{\Omega}_{\beta}, \boldsymbol{\Theta}_{t} \sqrt{\kappa}^{-1} \mathbf{S}_{R} \mathbf{X} \boldsymbol{\Omega}_{\alpha}\right) \\
& =\left(\mathbf{S}_{R} \mathbf{X} \boldsymbol{\Omega}_{\alpha}, \mathbf{S}_{R} \mathbf{Y}^{*} \mathbf{O}^{*}\left(d^{t}\right) \boldsymbol{\Omega}_{\beta}\right)=\left(k^{-1} h_{X}\left(\mathbf{X}^{*}\right) \boldsymbol{\Omega}_{\beta}, h_{Y}^{-1} h_{O}^{-1}\left(\mathbf{O}\left(d^{t}\right) \mathbf{Y}\right) \boldsymbol{\Omega}_{\alpha}\right) .
\end{aligned}
$$

In the last expression we extract the character phases using (2.13) and then exchange the order of $\mathbf{X}$ and $\mathbf{O}\left(d^{t}\right)$ using (2.14). Reinserting into (4.19) results in the following identity

$$
\eta\left(\mathbf{O}^{*}\left(d^{t}\right) \mathbf{X}^{*} \boldsymbol{\Omega}_{\beta}, \mathbf{Y} \boldsymbol{\Omega}_{\alpha}\right)=\eta\left(\boldsymbol{\Omega}_{\beta}, \mathbf{O}\left(d^{t}\right) \mathbf{X} \mathbf{Y} \boldsymbol{\Omega}_{\alpha}\right)=\left(\mathbf{Y}^{*} \mathbf{O}^{*}\left(d^{t}\right) \boldsymbol{\Omega}_{\beta}, \mathbf{U}_{t}(2 \pi i) \mathbf{X} \boldsymbol{\Omega}_{\alpha}\right)
$$

where $\eta=\chi_{X O Y}\left(h_{X} k^{-1}\right) \delta_{X O}(-)$ is the accumulated phase, depending both on the statistics phases and the group characters of the involved kink operators. The first expression in 
(4.20) in particular shows that the $t \rightarrow \infty$ limit of these matrix elements exists and yields well-defined matrix elements between scattering states. Since $\mathbf{O}$ is a cone-localized operator each of the matrix elements is separately well-defined also for $d^{t}=0$. On the other hand $\eta$ depends (for given kink operators) only on the orientation of the interpolating automorphisms and in particular is independent of $d^{t}$. The identity (4.20) thus remains valid when sending $d^{t}$ to zero. Adopting the notation from (3.15), (3.3) and (4.16) one arrives at

$$
\begin{aligned}
\eta^{\text {in }}\left\langle\bar{\theta}_{n-k+1}-i \pi, \bar{a}_{n-k+1} ; \ldots ; \bar{\theta}_{n}-i \pi, \bar{a}_{n}|\mathcal{O}| \theta_{n-k}, a_{n-k} ; \ldots ; \theta_{1}, a_{1}\right\rangle^{\text {out }} \\
\quad=\eta^{\text {out }}\left\langle 0|\mathcal{O}| \theta_{n}, a_{n} ; \ldots ; \theta_{1}, a_{1}\right\rangle^{\text {out }} \\
\quad={ }^{\text {out }}\left\langle 0|\mathcal{O}| \theta_{n-k}, a_{n-k} ; \ldots ; \theta_{1}, a_{1} ; \theta_{n}+i 2 \pi, a_{n} ; \ldots ; \theta_{n-k+1}+i 2 \pi, a_{n-k+1}\right\rangle^{\text {out }}
\end{aligned}
$$

for ordered and separated rapidities, i.e. $\theta_{j}-\theta_{j+1}>\epsilon, j=1, \ldots, n-1$ with some positive constant $\epsilon$. Both the "crossing relation" and the "cyclic form factor equation" are special cases of (4.21). For example one has

$$
\begin{aligned}
& \operatorname{in}\left\langle\bar{\theta}_{n}, \bar{a}_{n}|\mathcal{O}| \theta_{n-1}, a_{n-1} ; \ldots ; \theta_{1}, a_{1}\right\rangle^{\text {out }}={ }^{\text {out }}\left\langle 0|\mathcal{O}| \theta_{n}+i \pi, a_{n} ; \ldots ; \theta_{1}, a_{1}\right\rangle^{\text {out }} \\
& { }_{\text {out }}\left\langle 0|\mathcal{O}| \theta_{n-1}, a_{n-1} ; \ldots ; \theta_{1}, a_{1} ; \theta_{n}+2 \pi i, a_{n}\right\rangle^{\text {out }}=\eta^{\text {out }}\left\langle 0|\mathcal{O}| \theta_{n}, a_{n} ; \ldots ; \theta_{1}, a_{1}\right\rangle^{\text {out }}
\end{aligned}
$$

Analogues with 'in' and 'out' scattering states exchanged follow from (4.16).

The purpose of this paper was to provide a quantum field theoretical derivation of the cyclic form factor equation (1.1) or (4.21). The derivation given shows that it is a generic feature - not tied to integrability - of massive $1+1 \mathrm{dim}$. QFTs with a proper relativistic scattering theory. Keeping this in mind, we propose retaining the term "cyclic form factor equation" for it. The main technical tool in the derivation was the use of a family of Rindler spacetimes $t \rightarrow R^{t}$, comoving with the essential support regions of the interpolating quantum fields, to transfer the action of geometric modular structures to scattering states. We expect that a 4-dim. counterpart of the cyclic form factor equation can be derived along similar lines, to which we intend to return elsewhere.

Acknowledgements: I wish to thank H.-J. Borchers and K.-H. Rehren for discussions, as well as B. Schroer for correspondence and a stimulus [17 to write this up. In particular I am indebted to K.-H. Rehren for clarifing much of the material of section 2. The author acknowledges support by the Reimar Lüst fellowship of the Max-Planck-Society. 


\section{References}

[1] Smirnov, F.A.: A general formula for soliton form factors in the quantum SineGordon model, J. Phys. A19 (1986) L575-L578.

[2] Smirnov, F.A.: Form Factors in Completely Integrable Models of Quantum Field Theory, World Scientific, 1992.

[3] Karowski, M. and Weisz, P.: Exact form factors in 1+1 dim. field theoretical models with soliton behaviour, Nucl. Phys. 139 (1978) 455-476.

[4] Bisognano, J. and Wichmann, E.: On the duality condition for a hermitian scalar field, J. Math. Phys. 16 (1975) 985-1007.

Bisognano, J. and Wichmann, E.: On the duality condition for quantum fields, J. Math. Phys. 17 (1976) 303-321.

[5] Borchers, H.-J.: The CPT theorem in 2-dim. theories of local observables, Comm. Math. Phys. 143 (1992) 315-332.

[6] Haag, R., Hugenholtz, W. and Winnink, M.: On the equilibrium states in quantum statistical mechanics, Comm. Math. Phys. 5 (1967) 215-236.

[7] Takesaki, M.: Tomita's theory of modular Hilbert algebras and its application, Lecture Notes in Mathematics, Springer 1970.

[8] Bratteli, O. and Robinson, D.: Operator Algebras and Quantum Statistical Mechanics, Vol. 1,2; 2nd ed., Springer 1987.

[9] Haag, R.: Quantum field theories with composite particles and asymptotic conditions, Phys. Rev. 112 (1958) 669

Ruelle, D.: On the asymptotic condition in quantum field theory, Helv. Phys. Acta 35 (1962) 147.

[10] Haag, R.: Local Quantum Physics, 2nd ed., Springer 1996.

[11] Doplicher, S., Haag, R. and Roberts, J.: Local observables and particle statistics I, II, Comm. Math. Phys. 23 (1971) 199-230 and 35 (1974) 49-85.

[12] Buchholz, D. and Fredenhagen, K.: Locality and the structure of particle states, Comm. Math. Phys. 84 (1982) 1. 
[13] Fröhlich, J.: New super-selection sectors (soliton states) in 2-dim bose quantum field models, Comm. Math. Phys. 47 (1976) 269-310.

[14] Fredenhagen, K.: Superselection sectors in low dim. QFT, J. Geom. Phys. 11 (1993) 337.

[15] Schlingemann, D.: On the algebraic theory of kink sectors: Application to quantum field theory models and collision theory, PhD thesis 1996, DESY-96-228.

[16] Müger, M.: Superselection structure of massive QFTs in 1+1 dim., DESY 97-081, hep-th/9705019.

[17] Schroer, B.: Modular localization and the bootstrap-form factor program, Nucl. Phys. B499 (1997) 547.

[18] Rehren, K.-H.: Spin-Statistics and CPT for Solitons, preprint in preparation. 